\title{
Depletion of the Photosystem II Core Complex in Mature Tobacco Leaves Infected by the Flavum Strain of Tobacco mosaic virus
}

\author{
Kirsi Lehto, Mikko Tikkanen, Jean-Baptiste Hiriart, Virpi Paakkarinen, and Eva-Mari Aro \\ Department of Plant Physiology and Molecular Biology, University of Turku, FIN-20014 Turku, Finland
}

Submitted 19 February 2003. Accepted 30 July 2003.

\begin{abstract}
The flavum strain of Tobacco mosaic virus (TMV) differs from the wild-type (wt) virus by causing strong yellow and green mosaic in the systemically infected developing leaves, yellowing in the fully expanded leaves, and distinct malformations of chloroplasts in both types of infected tissues. Analysis of the thylakoid proteins of flavum strain-infected tobacco leaves indicated that the chlorosis in mature leaves was accompanied by depletion of the entire photosystem II (PSII) core complexes and the 33-kDa protein of the oxygen evolving complex. The only change observed in the thylakoid proteins of the corresponding wt TMV-infected leaves was a slight reduction of the $\alpha$ and $\beta$ subunits of the ATP synthase complex. The coat proteins of different yellowing strains of TMV are known to effectively accumulate inside chloroplasts, but in this work, the viral movement protein also was detected in association with the thylakoid membranes of flavum strain-infected leaves. The mRNAs of different enzymes involved in the chlorophyll biosynthesis pathway were not reduced in the mature chlorotic leaves. These results suggest that the chlorosis was not caused by reduction of pigment biosynthesis, but rather, by reduction of specific proteins of the PSII core complexes and by consequent break-down of the pigments.
\end{abstract}

Additional keywords: D1 protein, photosynthetic electron transfer, tobamoviruses.

Chlorosis in plants is a generic stress response caused by many different biotic and abiotic factors; it also commonly occurs as a symptom of different viral diseases. The primary causes of chlorosis may vary widely but, in all cases, the ultimate visible phenotype is associated with reduced chlorophyll content of the leaves. In different virus diseases, the induction of chlorotic symptoms may be mediated by a variety of different virus-induced physiological disturbances and virus-host interactions but, in principle, all these should be related either to reduced chlorophyll biosynthesis or to enhanced degradation of pigments (Balachandran et al. 1997). Down-regulation of the synthesis of photosynthetic pigments and proteins can be induced either by alterations in carbon metabolism, partitioning or translocation, or disturbed assembly of the photosynthetic pigment-protein complexes. Assembly of the photosynthetic chlorophyll protein complexes takes place in a highly coordinated manner, in which translation of the chlorophyll-binding proteins is dependent on the availability of the pigment in the

Corresponding author: K. Lehto; Telephone: + 3582333 6266; Fax: +358 2333 5549; E-mail: klehto@utu.fi complex (Green and Durnford 1996; von Wettstein et al. 1995). Also the self-life of chlorophyll is likely to depend on the correct assembly of these photosynthetic protein complexes, and enhanced degradation of photosynthetic pigments may occur when one or more of the protein components are not available for the assembly of the pigment-protein complexes.

The virus-encoded gene products that affect the development of chlorotic symptoms have been intensively studied, using different strains of Tobacco mosaic virus (TMV). Analysis of several attenuated strains has indicated the virus-encoded helicase or replicase proteins as the inducer or inducers of chlorotic symptoms (Bao et al. 1996; Lewandowski and Dawson 1993; Shintaku et al. 1996). On the other hand, the viral coat protein (CP) is also known to strongly affect symptom development; mutants with specific truncations of the $\mathrm{CP}$ gene cause severe yellowing on inoculated mature leaves and malformation of the chloroplast ultrastructure (Dawson et al. 1988; Lindbeck et al. 1991, 1992). Also, some spontaneous mutant strains of wt TMV (e.g., the flavum strains and the YSI/1 strain) cause distinctly severe symptoms both in the young and the mature leaves of infected plants (Banerjee et al. 1995). Mutations contained within the CP of these strains lead to disturbed folding of these proteins and to strong accumulation of aggregated, denatured CP in the infected cells (Jockusch et al. 2001). These viruses accumulate in the tissues at a much lower level than wt TMV (Banerjee et al. 1995; Oxelfelt 1970), but their coat proteins accumulate effectively inside host cell chloroplasts and associate with the thylakoid membranes (Banerjee at al. 1995). Several early investigations suggested that the distinctly severe symptoms of these viruses would be related to the mere accumulation of the viral $\mathrm{CP}$ in the chloroplasts and to reduced photosynthetic electron transfer in virus-infected plants (Banerjee et al. 1995; Hodgson et al. 1989; Reinero and Beachy 1989), but other studies have shown that these phenomena are not directly correlated (Rahoutei et al. 2000; van Kooten et al. 1990).

A recent study (Abbink et al. 2002) showed that the 126-kDa helicase protein of wt TMV has high binding affinity for the 33$\mathrm{kDa}$ protein component of the oxygen evolving complex (OEC). TMV infection also reduces the mRNA of the $33-\mathrm{kDa}$ protein, as well as the mRNAs of some other chloroplast-targeted proteins, i.e., the small subunit of Rubisco and the Rieske protein of the cytochrome $b_{6} f\left(c y t b_{6} f\right.$ ) complex. This finding as well as the gene expression studies of Itaya and associates (2002) suggest that the TMV infection affects transcriptional regulation of several chloroplast-targeted proteins and that the reduced expression of specific host gene products might be related to the induction of chlorosis. However, no differences have so far been observed in the chloroplast protein patterns of TMV-infected plants as compared with healthy controls (Reinero and Beachy 1989). 
In this work, we have investigated different molecular and physiological alterations in the flavum D19A strain of TMV(hereafter called flavum strain of TMV) (Jockusch et al. 2001; Melchers 1942) infected mature and young tobacco leaves, in order to unravel the molecular mechanisms leading to the strong chlorotic and yellowing symptoms in these leaves, respectively. Specifically, we have aimed to determine whether the flavum strain-induced reduction of chlorophyll (chlorosis) is related to alterations of the protein components of the photosynthetic chlorophyll protein complexes or to possible changes in the transcriptional regulation of chloroplast-targeted proteins, which are involved in chlorophyll biosynthesis and stabilization.

\section{RESULTS}

\section{Disease symptoms.}

Inoculation of the fully expanded lower leaves of young tobacco plants with the flavum strain led to chlorotic mottling of the inoculated leaves and development of strong vein clearing in the upper expanding leaves at about 6 days after inoculation (p.i.). Within the next days, the vein clearing turned bright yellow and the newly growing upper leaves showed strong mosaic of dark green and yellow areas. The mature leaves below the systemically infected upper leaves also turned chlorotic as the infection spread down from the plant top. In the course of three weeks, the flavum strain-inoculated leaves turned necrotic, while the lowest leaves of wt TMV-inoculated plants showed only mild chlorosis. The amount of viral CP detected in the sodium dodecyl sulfate (SDS)-soluble leaf extracts of different flavum strain-infected leaves was always clearly (more than fivefold) lower than that detected in corresponding wt TMV infected leaves, and, in the dark green sectors of systemically infected upper leaves, the CP was barely detectable (data not shown).

Electron microscopical analysis of different leaf tissues of healthy and virus-infected plants at 12 days p.i. revealed that the cells in the yellow sectors of the upper leaves contained only very few, distinctly large chloroplasts. These were struc- turally similar to the chloroplasts in healthy plants with welldeveloped thylakoid membranes and grana stacks but often contained accumulations of unidentified granular material (Fig. 1A). Also, in dark green leaf sectors, the chloroplasts were structurally intact although with frequent large accumulations of starch granules (Fig. 1B), and they appeared similar in size and number to the chloroplasts in the corresponding healthy cells (Fig. 1E and F). Conversely, chloroplasts of the mature, chlorotic leaves showed multiple malformations. In general, they were swollen to nearly or fully globular form, the thylakoid membranes were distorted, and the grana were often missing, and most chloroplasts contained membrane-bound accumulations of unidentified material (Fig. 1C). These chloroplast malformations differed from those reported earlier for infections caused by TMV mutants with truncated CP in which the thylakoids were replaced by undifferentiated tubular membranes (Lindbeck et al. 1991, 1992). The chloroplast ultrastructure of the wt TMV-infected plants (Fig. 1D) appeared very similar to that of healthy control plants although again with large accumulations of starch and with a slightly reduced number of thylakoid membranes and grana stacks, as compared with healthy controls. Similar cloroplast ultrastrucutres of wt TMV-infected plants can also be seen in figures published by Esau (1968).

\section{Alterations in the thylakoid protein complexes of the flavum strain-infected plants.}

Intact chloroplasts were lysed to isolate the thylakoid membrane fraction as described below. Solubilized thylakoid proteins were first analyzed by two-dimensional (Bluenative/SDS-polyacrylamide gel electrophoresis (PAGE) to investigate alterations in macromolecular thylakoid protein complexes possibly associated with the observed symptoms and with the chloroplast malformations. This analysis revealed a complete depletion of the monomeric and dimeric photosystem II (PSII) complexes, seen in Figure 2 as the loss of D1, D2, $\mathrm{CP} 43$, and CP47 proteins of the PSII core dimers and monomers in the flavum strain-infected mature leaves. Other major

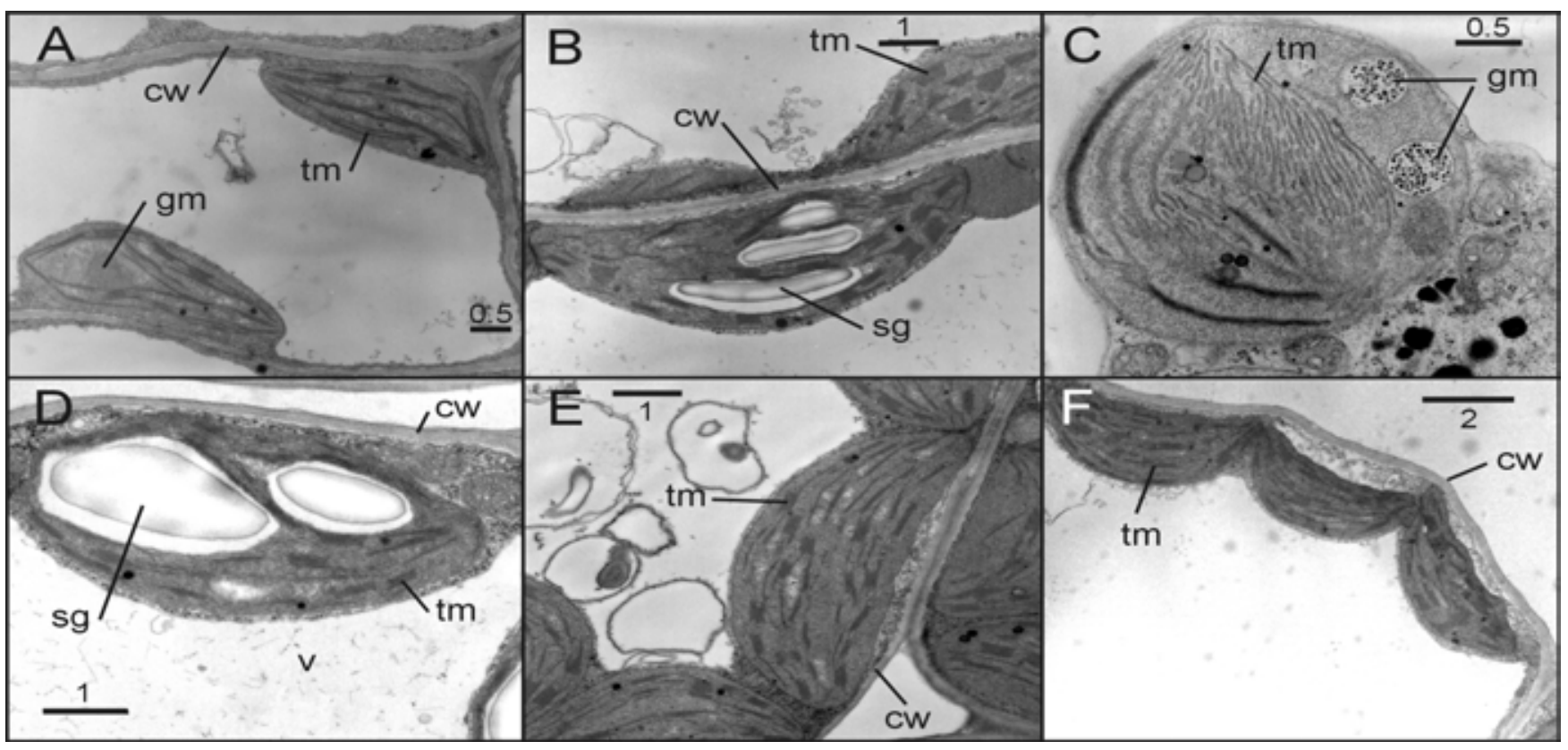

Fig. 1. Cytoplasmic structures and chloroplasts observed in the $\mathbf{A}$, light and $\mathbf{B}$, dark green sections of the top leaves and in the $\mathbf{C}$, mature chlorotic leaves of flavum strain-infected plants at 12 days after inoculation. Typical chloroplast of wild-type Tobacco mosaic virus-infected and healthy control leaves are shown in D, and E, respectively, and multiple chloroplasts aligning the cell walls in healthy cells are shown in F. The cell walls (cw), thylakoid membranes (tm), starch granules (sg), accumulations of unidentified granula matter (gm), and accumulations of virions (v) are indicated. The length of the scale bars in micrometers is shown next to each scale bar. 
thylakoid protein complexes, photosystem I (PSI), light-harvesting chlorophyll $a / b$ protein complex II (LHCII), ATP synthase, and the Cyt $\mathrm{b}_{6} \mathrm{f}$ complexes, were properly assembled and appeared unaffected by the infection (Fig. 2).

To visualize and compare the total thylakoid protein patterns of flavum strain- and wt TMV-infected plants at different stages of infection and leaf development, several leaf samples were collected at 20 days p.i. from the infected plants and from the corresponding leaves of healthy plants and were analyzed by SDS-PAGE and by Western blotting, using different antibodies. The symptoms and developmental stages of the three flavum strain-infected leaves were as follows: the lowest viable but chlorotic mature leaf, the subsequent upper, mildly chlorotic mature leaf, and the small top leaf showing severe mosaic of dark green and light areas. Coomassie blue-staining of the gel revealed depletion of the $\mathrm{CP} 47$ and $\mathrm{CP} 43$ proteins and of the 33-kDa protein component of the OEC from the thylakoids of the fully and of the mildly chlorotic mature leaves of the flavum strain-infected plants (Fig. 3A, lanes 1 and 2), whereas the thylakoid proteins from the upper leaves did not differ from those of the healthy control plants (Fig. 3A, lane 3). Western blot analysis using antiserum against the D1 protein revealed that this component of the PSII also was depleted in mature leaves showing different degrees of chlorosis (Fig. 3B, lanes 1 and 2). Together, the observed depletion of CP47, CP43, and D1 proteins of the PSII core complex and the 33$\mathrm{kDa}$ protein of OEC indicated that the individual components of PSII complexes did not occur even in nonassembled form in the thylakoid membrane. Western blot analysis using antiserum raised against the isolated PSI complex revealed no changes in the light-harvesting complex I (LHC I) polypeptides (Fig. 3C) nor in the PsaA or PsaB proteins of the PSI core complex (data not shown). In the Coomassie-stained gels, heavy new bands of 17.5- and 35-kDa proteins appeared in the thylakoid samples of the mature leaves of the flavum strain-infected plants (Fig. 3A, lanes 1 and 2), corresponding to the sizes of the viral $\mathrm{CP}$ monomer and dimer. By Western blotting, these were identified as viral $\mathrm{CP}$ (Fig. 3E). Only little viral $\mathrm{CP}$ was detected from thylakoids of young, developing leaves of the flavum straininfected plants (Fig. 3A and E, lane 3). The viral movement protein (MP) of $32 \mathrm{kDa}$ was detected by Western blotting in the thylakoid samples of only the mature leaves of the flavum strain-infected plants (Fig. 3F).

Polypeptide analysis of thylakoid membranes revealed small changes also in the thylakoid protein profile of the wt TMVinfected plant. The bands of 54- and $55-\mathrm{kDa}$ proteins, confirmed by immunoblotting as the $\alpha$ and $\beta$ subunits of the ATPsynthase, were reduced (Fig. 3A and D, lanes 1, 2, and 3 for wt TMV). These proteins apparently vary easily in different conditions, since they were reduced also in the upper leaves and, conversely, increased in the mature leaves of flavum straininfected plants, as compared with healthy controls (Fig. 3A and F, lanes 3, 1, and 2, respectively, for flavum strain TMV). Also in the wt TMV-infected leaves, a light band appeared in position of $17.5 \mathrm{kDa}$, corresponding to the viral coat protein (Fig. $3 \mathrm{~A}$ and E, lanes 2 and 3 for wt TMV). Viral MP (Fig. 3F, lanes

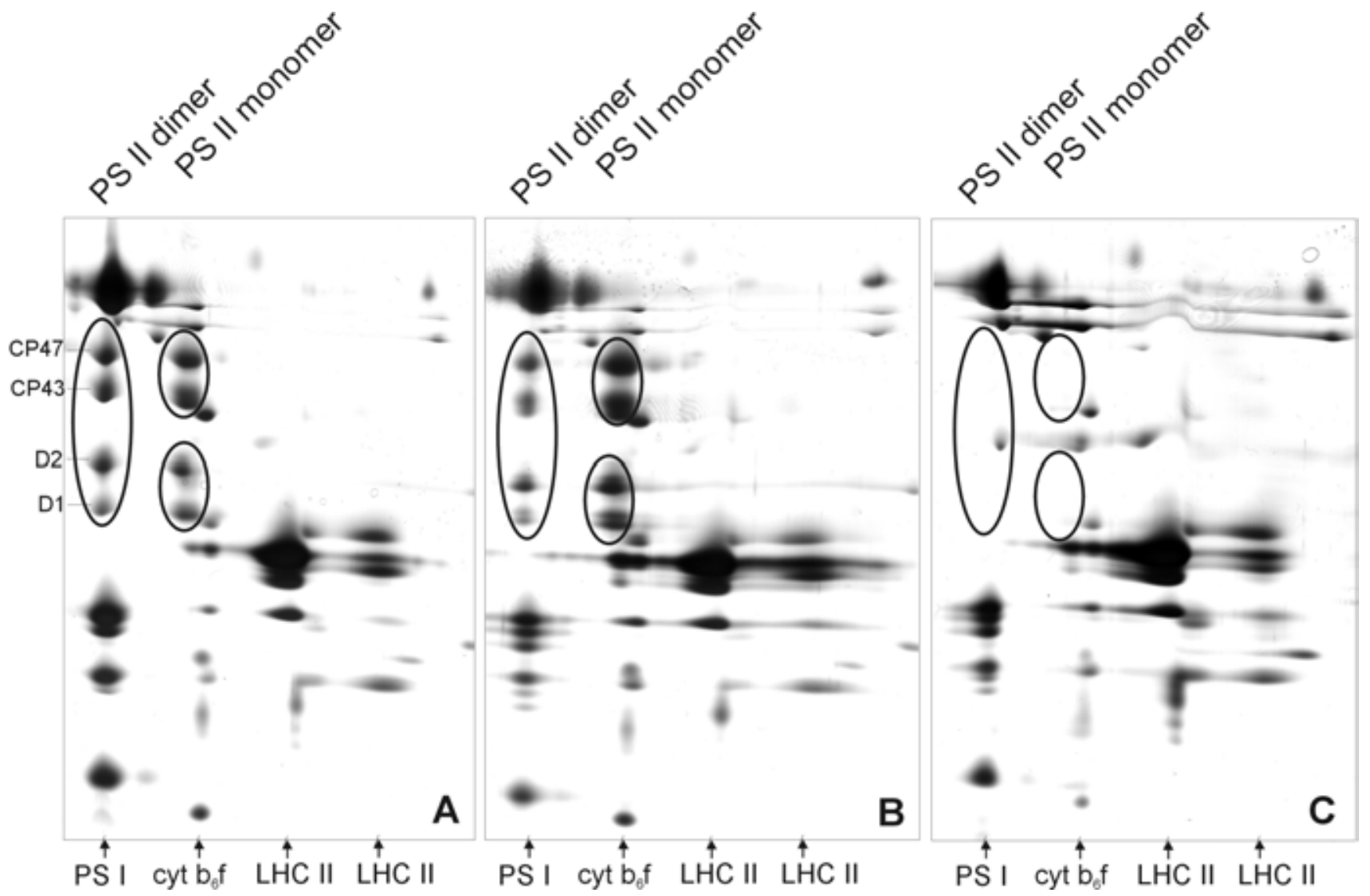

Fig. 2. Two-dimensional gel electrophoresis analysis of thylakoid protein complexes of A, leaves of healthy plants, B, corresponding flavum straininfected mature leaves at 20 days after inoculation, and leaves of $\mathbf{C}$ wild-type Tobacco mosaic virus-infected plants. Dodecyl-maltoside solubilized thylakoid protein complexes were first separated by blue-native gel electrophoresis (horizontal direction, with decreasing size of protein complexes from left to right), and then, the gel strip was subjected to second dimension sodium dodecyl sulfate-polyacrylamide gel electrophoresis analysis (vertical direction). Identification of the protein complexes is based on mass-spectrometry of trypsin-digested protein spots (Suorsa et al. 2001). The positions of the major core proteins D1, D2, CP43, and CP47 of the PSII dimer, PSII monomer, and CP43-less PSII monomer complexes are indicated by circles. Positions for the unaltered PSI, cyt $\mathrm{b}_{6} \mathrm{f}$, and LHCII (monomer and trimer) complexes are indicated by arrows. 
1, 2, and 3 for wt TMV) was not detected in the thylakoid samples of wt TMV-infected plants.

\section{Reduction of chlorophyll content} and PSII efficiency at different stages of infection.

During the progressing spread of virus infection, different stages of the infection can be detected in the different leaves of inoculated tobacco plants. Therefore, the upper, middle, and lower fully expanded leaves of flavum strain- and wt TMVinoculated plants were analyzed at different times after inocu- lation to determine how the progressing infection affects chlorophyll content, chlorophyll $a / b$ ratio, and PSII photochemical efficiency $\left(\mathrm{F}_{\mathrm{v}} / \mathrm{F}_{\mathrm{m}}\right)$ in these leaves. Table 1 shows the results of the analysis at 13 days p.i., at the time when infected plants had seven fully developed leaves, of which the two lowest, inoculated leaves and the three fully expanded upper leaves were chlorotic but the fourth and fifth leaves were still virtually symptomless. The $F_{v} / F_{m}$ value of the dark green tissues of the top leaves of flavum strain-infected plants was the same as in the corresponding leaves of healthy plants, although their chlo-

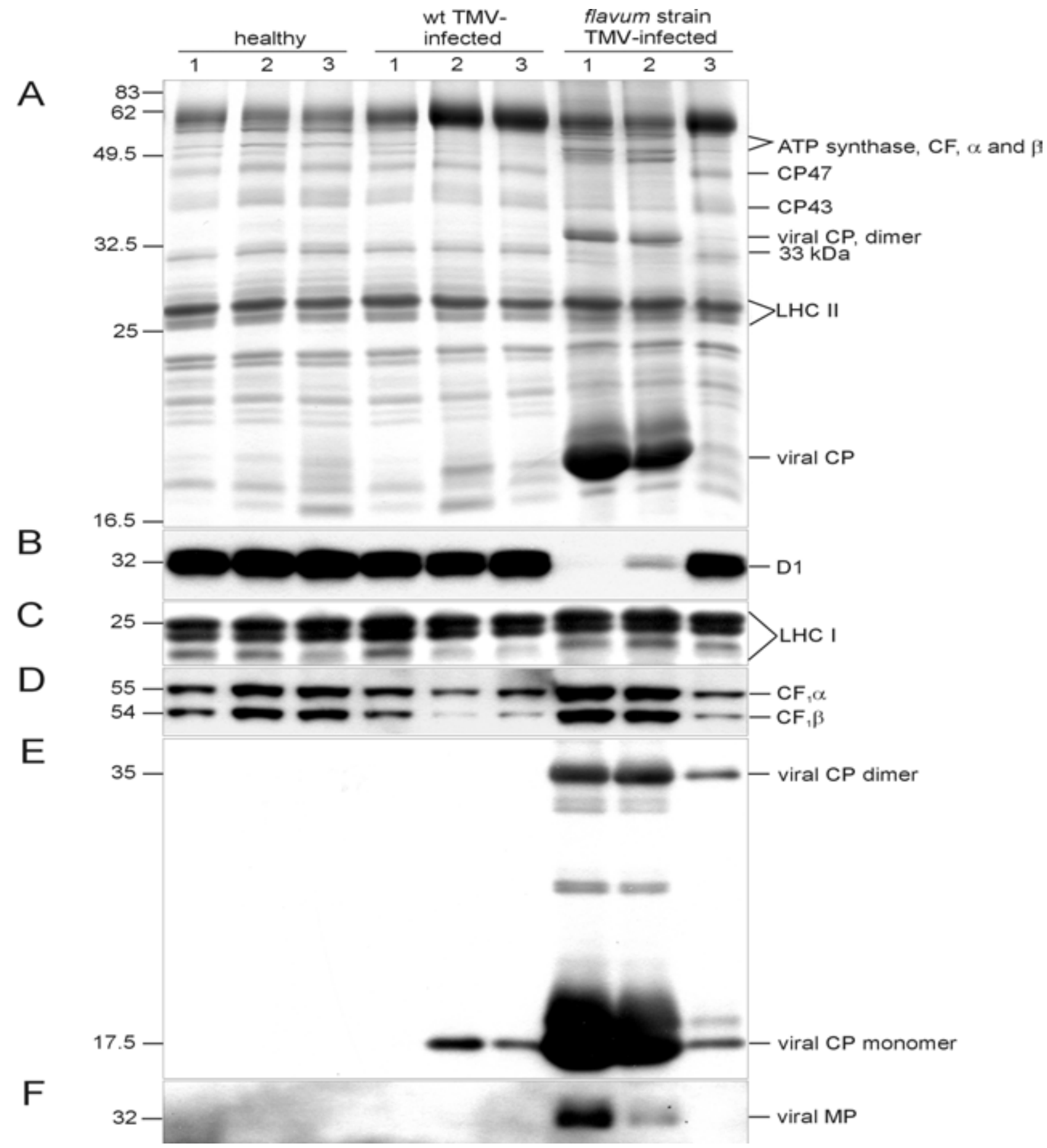

Fig. 3. Sodium dodecyl sulfate-polyacrylamide gel electrophoresis (SDS-PAGE) and Western blot analysis of the thylakoid protein samples of different leaves of flavum strain- and wild-type (wt) Tobacco mosaic virus (TMV)-infected and healthy plants at 20 days after inoculation. Samples (1 $\mu \mathrm{g}$ chl/ $20 \mu \mathrm{l})$ were loaded in the following order: lane 1, the lowest viable leaf with strong chlorosis; lane 2, the subsequent upper leaf with mild chlorosis; and lane 3 , the small top leaf with strong mosaic of the flavum strain-infected plant and the corresponding leaves of wt TMV-infected and healthy control plants, as indicated. A, Coomassie blue-stained SDS-PAGE gel with indications for the major protein bands. Western immunoblots of similar gels labeled with antibodies raised against $\mathbf{B}$, the photosystem II core protein D1, C, the photosystem I light harvesting proteins $(\mathrm{LHCI})$, and $\mathbf{D}, \mathrm{CF}_{1} \alpha$ and $\mathrm{CF}_{2} \beta$ components of the ATP synthase and against the viral proteins $\mathbf{E}$, TMV coat protein (CP) and F, TMV movement protein (MP). 
rophyll content was clearly reduced in comparison with the control plants (Table 1). In the yellow areas of the top leaves as well as in all the lower, chlorotic leaves of the flavum straininfected plants, both the chlorophyll content and the $\mathrm{F}_{\mathrm{v}} / \mathrm{F}_{\mathrm{m}}$ value were strongly reduced as compared with the corresponding leaves of healthy plants. The $F_{v} / F_{m}$ value was also slightly reduced in leaf 4 , which at 13 days p.i. was still fully green (Table 1). At 16 days p.i., both the chlorophyll content and the $\mathrm{F}_{\mathrm{v}} / \mathrm{F}_{\mathrm{m}}$ value were strongly reduced also in this leaf, although it did not show visible symptoms as yet (Table 1). SDS-PAGE and Western immunoblot analysis of the thylakoid samples of such nonsymptomatic middle leaves revealed that they contained a low level of viral CP and that the D1 protein and the $33-\mathrm{kDa}$ component of the OEC were reduced already prior to the appearance of chlorotic symptoms (data not shown).

\section{Accumulation of mRNAs of the key enzymes of the chlorophyll biosynthesis pathway and of the major chlorophyll-binding apoproteins.}

Reduction of transcripts of several chloroplast-targeted proteins has been observed in connection with tobamovirus infections (Abbink et al. 2002; Itaya et al. 2002). Inhibition of the chlorophyll biosynthesis pathway might occur associated with chlorotic virus symptoms, either as a cause of the chlorotic symptom or as a feed-back reaction caused by disturbed assembly of chlorophyll protein complexes. Therefore, we investigated whether flavum strain infection hampers the accumulation of mRNAs of the key enzymes involved in chlorophyll biosynthesis (Fig. 4) or of mRNAs of some key apoproteins involved in the binding and stabilization of newly synthesized chlorophyll.

Each target mRNA was analyzed using quantitative real time reverse transcription-polymerase chain reaction (RT-PCR) (discussed below) and using the $25 \mathrm{~S}$ ribosomal RNA as the internal standard for relative RNA quantification. The mRNAs were first quantified from healthy tobacco leaves and were found to vary widely, depending on leaf position for some but not for all of the target genes. In the youngest top leaves, the mRNA levels varied even between the different sampling sites within the leaf, i.e., between the tip and the base of the leaf (Fig. 5). Therefore, each target mRNA was analyzed from several different leaf positions in the infected plant, and particular attention was paid to analyze control samples from exactly corresponding positions in the healthy plant. Amounts of the different mRNAs of the healthy leaves and of corresponding flavum strain-infected leaves, analyzed side by side in parallel RT and PCR reactions and determined as means of three independent analyses of each sample, are indicated in Figure 5. The analyzed leaves were the young top leaf (L1) showing strong mosaic, separated into the bright yellow tissues in the tip of the leaf (L1T) and the dark green tissues in the base of the leaf (L1B), leaf 4 (L4), showing strong chlorosis with green veins, leaf 6 (L6), showing developing chlorosis with yellow veins, and leaf 7 (L7), showing full chlorosis. The experiments were repeated three times from independently grown sets of plants.

In the chlorophyll biosynthesis pathway, we investigated the putative transcriptional (mRNA accumulation) changes in the beginning of the pathway (glutamate-1-semialdehyde aminotransferase [GSA] and aminolevulinic acid dehydratase $[\mathrm{AlaD}]$ ), at the central branch point (ferrochelatase [FeChe] leading to the biosynthesis of heme and $\mathrm{Mg}$-chelatease [MgChe], composed of three subunits encoded by the ChlD, $\mathrm{ChlH}$, and ChlI genes and leading to the biosynthesis of chlorophyll), and at the end of the pathway (protochlrophyllide oxidoreductase [PorB] and chlorophyll synthetase [CS]), where feedback control can regulate the chlorophyll yield and allow coordinated assembly of the chlorophyll protein complexes (Grimm 1998).

The mRNAs of the enzymes occurring early in the chlorophyll biosynthesis pathway (GSA, AlaD, FeChe, and all subunits of the $\mathrm{MgChe}$ enzyme) were either slightly or strongly (up to eightfold for GSA in leaf 4, L4) up-regulated in all flavum strain-infected leaves (Fig. 5A), except for the dark green part in the base of leaf 1 (L1B), as compared with healthy plants. Increase of all target mRNAs was strongest in the chlorotic leaf 4 (L4) and in the bright yellow sectors in the tip of leaf one (L1T). In the dark green tissue occurring in the base of leaf one (L1B), all the target mRNAs were either strongly (40 and $50 \%$ for $\mathrm{AlaD}$ and $\mathrm{ChlH}$, respectively) or slightly reduced compared with healthy leaves. The mRNA contents of both PorB and CS enzymes were not clearly affected or were only slightly reduced (less than $20 \%$ ) by infection in the mature leaves. In the top leaves also, these mRNAs were at a higher level in the yellow tissues in the tip of the leaves (L1T)

Table 1. Chlorophyll content ${ }^{\mathrm{a}}$

\begin{tabular}{|c|c|c|c|c|}
\hline Plant & Leaf position and symptom type & Total Chl & Chl $a /$ Chl $b$ & $\mathbf{F}_{\mathbf{v}} / \mathbf{F}_{\mathbf{m}}$ \\
\hline \multirow[t]{6}{*}{ Healthy } & Top leaves & $28.09 \pm 3.60$ & $4.59 \pm 0.10$ & $0.818 \pm 0.012$ \\
\hline & Half-expanded leaves & $28.27 \pm 0.79$ & $4.52 \pm 0.03$ & $0.836 \pm 0.002$ \\
\hline & 1st expanded leaves & $26.68 \pm 0.40$ & $4.47 \pm 0.01$ & $0.821 \pm 0.020$ \\
\hline & 3rd expanded leaves & $24.27 \pm 0.69$ & $4.33 \pm 0.18$ & $0.831 \pm 0.001$ \\
\hline & 4th expanded leaves & $24.22 \pm 1.17$ & $4.15 \pm 0.02$ & $0.816 \pm 0.010$ \\
\hline & Lower leaves & $14.05 \pm 1.15$ & $3.78 \pm 0.15$ & $0.778 \pm 0.023$ \\
\hline \multirow[t]{6}{*}{ Wild-type TMV infected } & Top leaves, with mosaic & $21.32 \pm 5.15$ & $4.74 \pm 0.24$ & $0.847 \pm 0.010$ \\
\hline & Half-expanded leaves & $14.99 \pm 0.92$ & $4.44 \pm 0.01$ & $0.766 \pm 0.017$ \\
\hline & 1st expanded leaves, chlorotic & $14.31 \pm 1.38$ & $4.37 \pm 0.06$ & $0.769 \pm 0.020$ \\
\hline & 3rd expanded leaves, green & $22.22 \pm 0.51$ & $4.30 \pm 0.13$ & $0.761 \pm 0.003$ \\
\hline & 4th expanded leaves, green & $18.35 \pm 0.61$ & $4.13 \pm 0.19$ & $0.749 \pm 0.009$ \\
\hline & Lower leaves, inoculated, green & $10.22 \pm 0.53$ & $3.61 \pm 0.13$ & $0.564 \pm 0.089$ \\
\hline \multirow[t]{8}{*}{ Flavum strain infected } & Top leaves, green & $16.00 \pm 1.27$ & $4.29 \pm 0.18$ & $0.832 \pm 0.008$ \\
\hline & Top leaves, yellow & $4.26 \pm 0.47$ & $3.15 \pm 0.25$ & $0.202 \pm 0.037$ \\
\hline & Half-expanded leaves, with strong vein-clearing & $5.14 \pm 1.31$ & $3.20 \pm 0.15$ & $0.195 \pm 0.045$ \\
\hline & 1st expanded leaves, strongly chlorotic & $6.68 \pm 1.25$ & $3.07 \pm 0.10$ & $0.139 \pm 0.041$ \\
\hline & 3rd expanded leaves, mildly chlorotic & $14.92 \pm 1.39$ & $3.63 \pm 0.13$ & $0.119 \pm 0.013$ \\
\hline & 4th expanded leaves, green & $19.22 \pm 0.77$ & $3.91 \pm 0.08$ & $0.762 \pm 0.086$ \\
\hline & 4th expanded leaves, green, at 16 days p.i. & $16.82 \pm 0.49$ & $3.94 \pm 0.11$ & $0.341 \pm 0.183$ \\
\hline & Lower leaves, inoculated, chlorotic & $8.43 \pm 0.59$ & $3.08 \pm 0.14$ & $0.084 \pm 0.055$ \\
\hline
\end{tabular}

\footnotetext{
${ }^{a}$ Chlorophyll $(\mathrm{Chl}) a / b$ ratio and photosystem II photochemical efficiency $\left(\mathrm{F}_{\mathrm{v}} / \mathrm{F}_{\mathrm{m}}\right)$ of different leaves of healthy plants and plants infected with either wild type or flavum strain Tobacco mosaic virus (TMV) at 13 days after inoculation (p.i.). The analysis of the developing infection in the fourth leaf of the flavum strain-infected plant was continued on 16 days p.i. The values are the means of three independent measurements from each target leaf, and the measurements have been repeated several times with very similar results.
} 
and were reduced in the dark green tissues in the base of the leaves (L1B) compared with healthy control leaves (Fig. 5 A).

Accumulation of the mRNAs of the nuclear-encoded Lhcbl and Lhcb2 genes, coding for the Lhcb1 and Lhcb2 apoproteins of LHCII, and of the chloroplast-encoded $p s b A$ gene, coding for the D1 protein component of the PSII reaction center, were also determined using quantitative RT-PCR (Fig. 5B). The mRNAs of both the Lhcb1 and Lhcb2 genes were not significantly affected by the infection in the mature leaves, but in the top leaf, both in the tip (yellow) and the base (dark green) tissues, these mRNAs were clearly ( 25 to $50 \%$ ) reduced, as compared with healthy controls. However, the mRNA of the $p s b A$ gene was about twofold higher in the yellow sectors of the top leaf of the flavum straininfected plants, while in the green sectors, this mRNA was reduced compared with healthy controls. In all mature, chlorotic leaves, the $p s b A$ mRNA level was remarkably higher (six to twofold) than in corresponding healthy leaves.

\section{DISCUSSION}

The chlorotic symptoms caused by different TMV strains or mutants are known to be determined either by the heli-

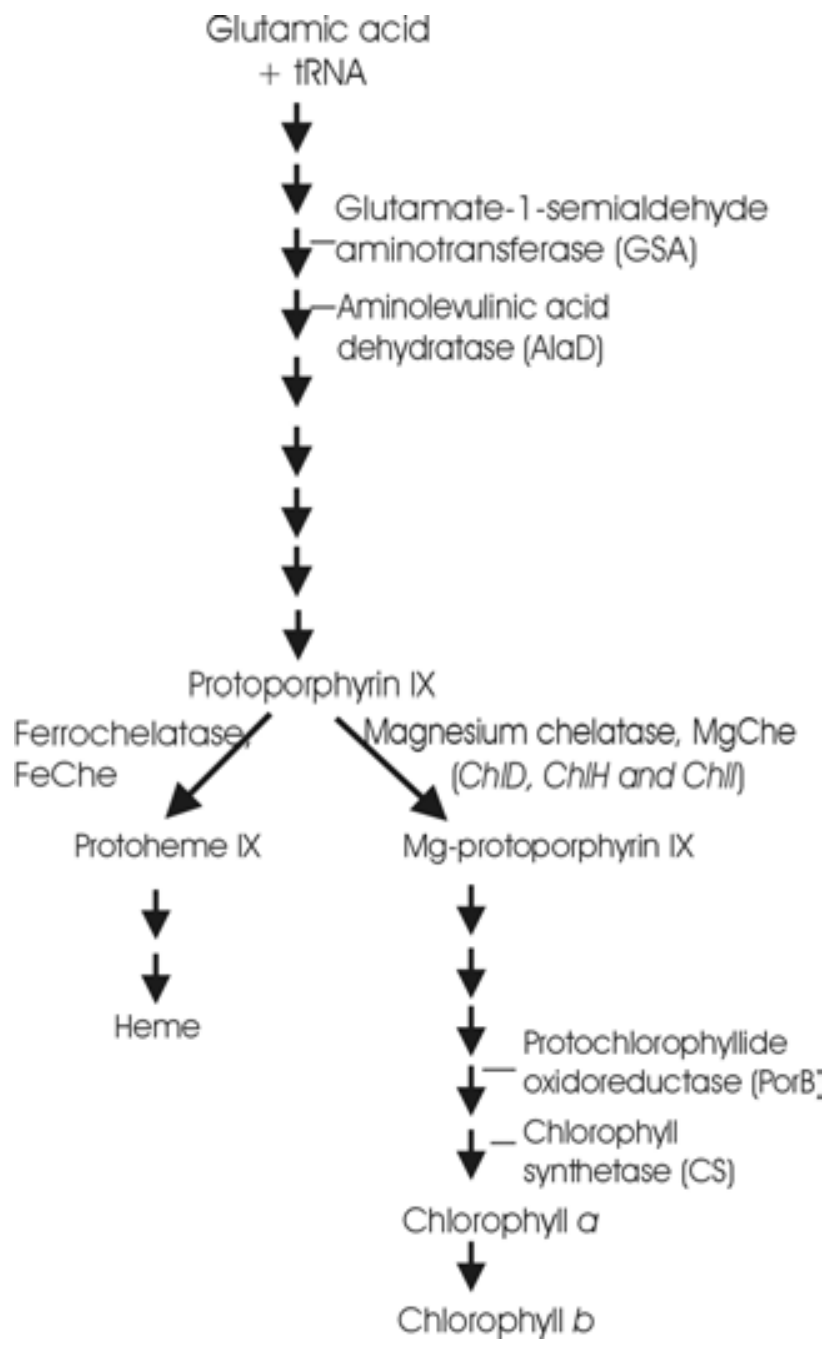

Fig. 4. Schematic presentation of the chlorophyll biosynthesis pathway. Abbreviations: GSA = glutamate-1-semialdehyde aminotransferase; $\mathrm{AlaD}=$ aminolevulinic acid dehydratase; FeChe = ferrochelatase, leading to the biosynthesis of heme; $\mathrm{MgChe}=$ magnesium chelatase, and $\mathrm{ChlH}$, ChlD, and ChlI $=$ the three subunits of $\mathrm{MgChe}$, leading to the biosynthesis of chlorophyll; PorB = protochlrophyllide oxidoreductase; $\mathrm{CS}=$ cholorophyll synthetase. Adopted according to Buchanan and associates (2000). case/replicase proteins (Bao et al. 1996; Shintaku et al. 1996) or by the coat protein (Culver et al. 1991). Also, different physiological disturbances are known to be caused by different tobamoviruses (Abbink et al. 2002; Rahoutei et al. 2000), and different chloroplast malformations are caused by the wt TMV and by various viral strains or mutants (Esau 1968; Lindbeck et al. 1991, 1992; this study). These differences suggest that, in each case, the chlorotic phenotype can be induced by different mechanisms and by different virus-host interactions. Indeed, Lindbeck and associates (1992) suggested that the severe chlorosis caused by some TMV CP deletion mutants is manifested on top of the milder chlorosis caused by the viral replicase proteins. In this work, we have investigated the physiological and molecular disturbances that co-occur in tobacco host with the strong yellowing and chlorotic symptoms caused by the flavum strain of TMV. The most striking physiological disturbance detected in mature, chlorotic leaves was the specific alteration of the thylakoid protein composition, i.e., the depletion of all the major protein components of the PSII core complex. This plant response was specific for the flavum strain, since no changes were observed in the thylakoid proteins of plants infected with four different strains of TMV (U1, U2, U5, and the recombinant virus strain TMV-30b) (Shivprasad et al. 1999; data not shown), except for a slight reduction of the $\alpha$ and $\beta$ subunits of the ATP synthase complex observed in the wt TMV infection (Fig. 3). The depletion of the PSII core complex was initiated prior to the appearance of the chlorotic symptoms, suggesting that it was causally related to the induction of chlorosis. Chlorosis was not caused by transcriptional down-regulation of chlorophyll biosynthesis or of the chlorophyll-stabilizing apoproteins, since the mRNAs of the key proteins related to these functions were not significantly affected or were increased in the chlorotic leaves, as compared with corresponding healthy leaves.

In healthy plants, the D1 protein component of PSII turns over exceptionally rapidly, due to light-induced inactivation and subsequent oxidative damage of PSII (Andersson and Aro 2001; Zhang and Aro 2002). In mature leaves, the chloroplastencoded D1 protein is thus by far the most rigorously synthesized thylakoid protein. Due to the central position of the D1 protein in the PSII pigment-protein complex, disruptions in D1 protein synthesis cause a complete disassembly and degradation of PSII complexes. This, in turn, enhances the production of reactive oxygen species and the breakdown of photosynthetic pigments. In flavum strain-infected mature leaves, the chlorotic phenotype was associated with nearly complete depletion of the D1 protein (Figs. 2 and 3). Interestingly, this was associated with a significant increase of the D1 protein mRNA (Fig. 5B), indicating that the plastid gene expression system attempted to compensate for the loss of the D1 protein at the transcriptional level. Consequently, the ultimate cause for D1 depletion should be either reduced translation or strongly enhanced degradation of the protein. Previous studies (Oxelfelt 1971) have shown a severe reduction of the chloroplast $23 \mathrm{~S}$, $16 \mathrm{~S}$, and $13 \mathrm{~S}$ rRNAs in flavum (D19A) strain-infected plants, suggesting that the loss of the D1 protein is related to the reduction of the protein translation capacity in organello. If this is the case, the reduction is specific for the flavum strain of TMV, since the maintenance of the PSII protein complexes was not affected in the wt TMV infections. Likewise, no reduction has been observed in the protein translation capacity of chloroplasts of wt TMV-infected plants or of chloroplasts of plants infected by a tobamovirus mutant TMV-PV230, which also causes severe symptoms (Reinero and Beachy 1989).

Thylakoid proteins from the bright yellow areas of systemically infected upper leaves were not analyzed separately, because these areas occurred as dense mosaic with the dark green tissues. The thylakoid protein profiles (samples adjusted on the 
basis of the chlorophyll content) obtained from the combined green and light tissues (Fig. 3) most likely presented only the green areas, since these contained several-fold more chloroplasts than the white areas. Other analysis, however, revealed specific disturbances in the bright yellow tissues. The ultrastructure of the chloroplasts in these tissues was fairly normal (Fig. 1), but the number of the chloroplasts was very low, and the chloroplasts were distinctly large, suggesting that the infection strongly disturbed the division but not the development of the plastids in the young leaves. This differs from the chloroplast structural disturbances caused by the artificial CP-deletion mutant cp35-5 (Lindbeck et al. 1992), which appeared to stop the development of the plastids at the proplastid stage but reportedly did not affect their number. It is conceivable that the prevention of chloroplast division in vigorously expanding leaves at least partially reflected the same process or processes, i.e., lack of proper synthesis and assembly of the thylakoid proteins, that led to depletion of the PSII core complexes in mature leaves.

In the yellow parts of the top leaf (L1T), the mRNA contents of most of the chlorophyll biosynthesis-related enzymes were greater than in corresponding tissues of healthy control plants, indicating that the development of chlorosis was not caused by transcriptional down-regulation of the chlorophyll biosynthetic pathway. However, a reduction of the Lhcbl and Lhcb2 mRNAs in these tissues is in accordance with the transcrip-
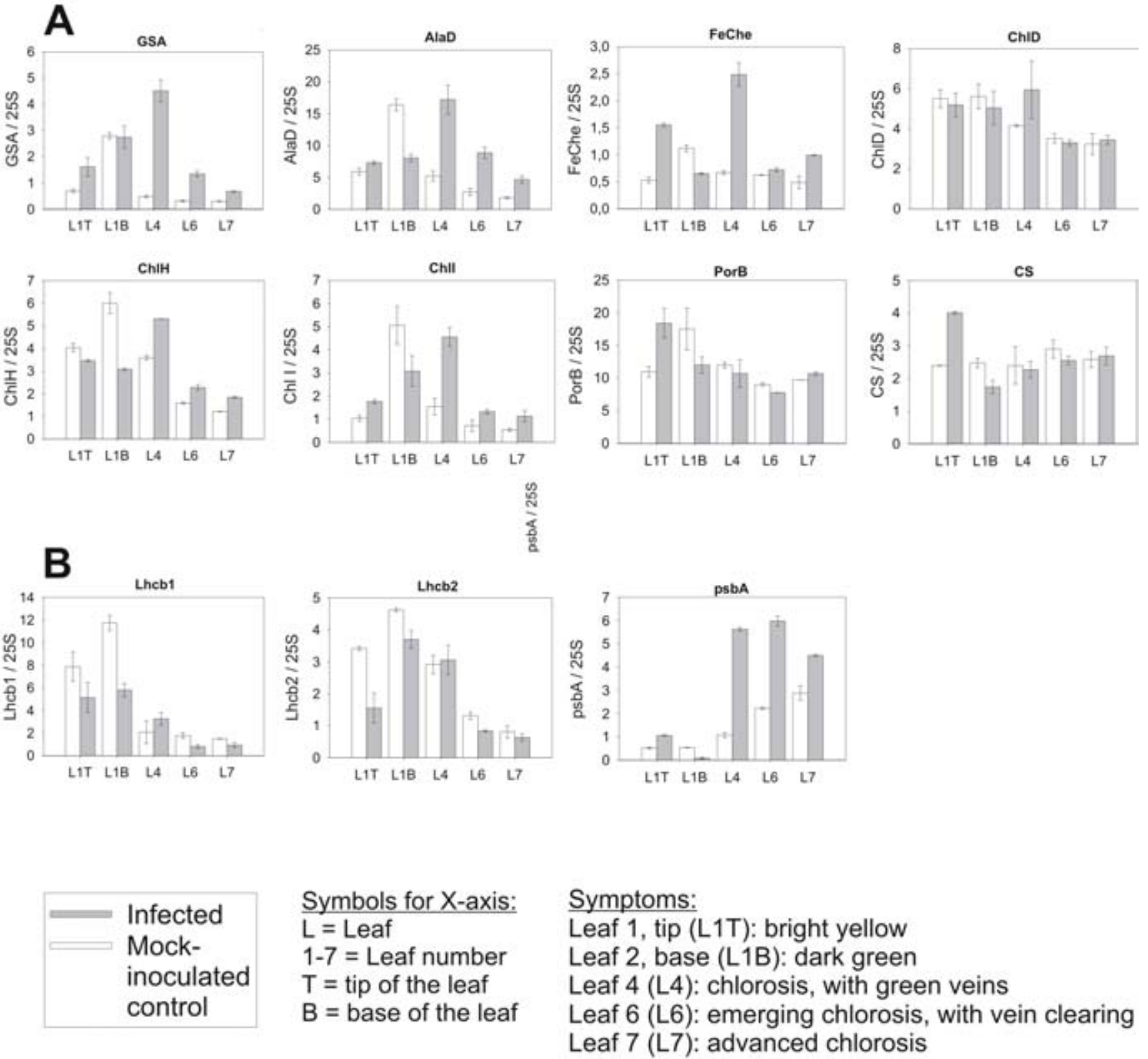

Fig. 5. Relative mRNA contents of selected chloroplast-targeted proteins in different leaves of flavum strain-infected and healthy tobacco plants, determined as means of three independent analyses of each sample, with the standard deviations indicated by error bars. A, mRNA levels of the key enzymes of the chlorophyll biosynthesis pathway. Abbreviations: GSA = glutamate-1-semialdehyde aminotransferase; AlaD = aminolevulinic acid dehydratase; FeChe $=$ ferrochelatase, leading to the biosynthesis of heme; $\mathrm{MgChe}=$ magnesium chelatase, and ChlH, ChlD, and ChlI = the three subunits of $\mathrm{MgChe}$, leading to the biosynthesis of chlorophyll; PorB = protochlrophyllide oxidoreductase; CS = cholorophyll synthetase. B, mRNA levels of the Lhcb1, Lhcb2, and $p s b A$ genes, coding for the Lhcb1, Lhcb2, and D1 protein components of photosystem II. All mRNA species were determined by quantitative reverse transcription-polymerase chain reaction from the same leaf total-RNA samples, isolated either from different leaves of healthy plants or from corresponding leaves of flavum strain-infected tobacco plants. All mRNAs were normalized to the $25 \mathrm{~S}$ ribosomal RNA level of the same sample. Symptoms of different leaves are described in the figure. 
tional down-regulation of distinct chloroplast-targeted proteins reported to occur in wt TMV infected plants (Abbink et al. 2002; Itaya et al. 2002). Interestingly, all the analyzed mRNAs were strongly reduced in the dark green sections of upper leaves although these tissues remained nearly free of the virus, apparently due to a silencing-mediated defense mechanism (Moore et al. 2001). However, the chlorophyll level in these tissues was only slightly reduced, and no changes were observed in the thylakoid protein profiles, as compared with healthy leaves. Thus, the transcriptional down-regulation observed both for the nuclear and chloroplast ( $p s b A$ )-encoded genes (Fig. 5) was not reflected in the accumulation of the thylakoid pigment-protein complexes. This indicates that the downstream regulatory mechanisms of different protein biosynthesis and turnover processes can compensate for the observed alterations at the mRNA level.

In accordance with the earlier results obtained with mutant YSI/1 (Banerjee et al. 1995), the induction of chlorotic symptoms should be related to the single mutation (A19D) also in the flavum strain CP. The CP of the flavum strain also accumulates inside chloroplasts in association with the thylakoid membranes much more effectively than the $\mathrm{CP}$ of $\mathrm{wt}$ TMV, in accordance with different yellowing-type mutant strains of TMV (Banerjee et al. 1995; Reinero and Beachy 1986). However, some doubt still exists as to whether the chlorotic symptoms would be related to the $\mathrm{CP}$ accumulation inside chloroplasts or to the strong accumulation of the $\mathrm{CP}$ as insoluble aggregates in the host cell cytoplasm, reported for the flavum and Ni118 strains (Jockusch et al. 2001) and for several artificial TMV mutants coding for truncated CPs (Lindbeck et al. 1991, 1992). Our data indicate that, at least in the mature leaves, the accumulation of the flavum strain $\mathrm{CP}$ inside the chloroplasts is significant because it disturbs the rapid turnover of the chloroplast-encoded D1 protein. However, in the white sections of the upper developing leaves, with a low number of chloroplasts, the accumulation of the denatured CP in the cytoplasm may also be significant. It is conceivable that, in such young leaves, the denatured (misfolded) CP in the host cytoplasm may disturb chloroplast development and function by interfering with the expression or transport of the multiple nuclear-encoded chloroplast proteins (Dawson 1992). These effects could be either direct or indirect, mediated, e.g., via induced expression of hostencoded heat shock 70 protein (Jockusch et al. 2001), which also functions as an essential chaperone of the chloroplasttargeted proteins (Jarvis and Soll 2001).

In this work, the flavum-strain encoded MP also was detected in association with the thylakoid membranes. Whether this observation has any physiological significance, e.g., in the exceptionally efficient accumulation of CP inside chloroplasts, remains to be investigated.

In conclusion, the chlorotic symptoms in the flavum straininfected mature tobacco leaves were not related to reduced accumulation of mRNAs of the enzymes or apoproteins involved in the biosynthesis or stabilization of chlorophyll. Rather, the induction of chlorotic symptoms was related to the accumulation of viral CP inside chloroplasts and in association with thylakoid membranes, to the depletion of the PSII core complexes, and to consequent production of reactive oxygen species. All these defects can be related to reduced D1 protein translation in chloroplasts due to the fact that the D1 protein is the only thylakoid component in mature leaves that requires constant synthesis and cotranslational insertion into the PSII complex (Zhang et al. 1999, 2001). In the vigorously expanding young leaves, additional mechanisms derived from cytoplasmic malfunctions may also be involved in the development of flavum strain-induced severe chlorosis.

\section{MATERIALS AND METHODS}

\section{Plant and virus material.}

The flavum strain of TMV was supplied by P. Oxelfelt and originates from Wittmann (Oxelfelt 1970). Inoculum for the wt TMV was transcribed from the infectious clone pTMV304, a T7 promoter-controlled derivative of the infectious clone pTMV204 (Dawson et al. 1986) obtained from W. O. Dawson. All experiments were performed on Nicotiana tabacum L. 'Xanthi' nn hosts. Mechanical inoculations of the fully expanded lower leaves dusted with carborundum were done by rubbing with leaf sap extracted with $50 \mathrm{mM} \mathrm{Na}$ phosphate buffer, $\mathrm{pH} 7.0$, from frozen, virus-infected leaves. Plants were grown at $22^{\circ} \mathrm{C}$ and with a $16 \mathrm{~h}$ light and $8 \mathrm{~h}$ dark period. The plants inoculated for the analysis of the thylakoid proteins were about five weeks old with three to five fully expanded leaves. The plants inoculated for the analysis of the photosynthetic parameters and for the quantitation of various mRNAs were about seven weeks old with five to seven fully expanded leaves.

\section{Electron microscopy.}

For electron microscopy, leaf sections were fixed with $3 \%$ glutaraldehyde in $0.1 \mathrm{M}$ Na-phosphate buffer, $\mathrm{pH} 7.0$, and postfixed in $1 \%$ osmium tetroxide in the same buffer. After dehydration in an alcohol series, the samples were embedded in Epon. Thin sections cut with KB ultramicrotome IV were stained with uranyl acetate and Reynolds lead citrate and were examined with a Jeol Jem 1200EX electron microscope.

\section{Isolation of thylakoid membranes.}

After removal of the midribs, tobacco leaves were homogenized in $330 \mathrm{mM}$ sorbitol, $50 \mathrm{mM}$ HEPES-KOH, pH 7.6, 2 $\mathrm{mM}$ EDTA, $1 \mathrm{mM} \mathrm{MgCl} 2,1 \%$ bovine serum albumin, were filtered through Miracloth, and were centrifuged for $5 \mathrm{~min}$ at $2500 \times g$ to pellet the intact chloroplasts. The pellets were resuspended in $5 \mathrm{mM}$ sorbitol, $50 \mathrm{mM}$ HEPES-KOH, pH 7.6, 5 $\mathrm{mM} \mathrm{MgCl}$, were centrifuged for $5 \mathrm{~min}$ at $3500 \times \mathrm{g}$, and finally, were resuspended in $100 \mathrm{mM}$ sorbitol, $50 \mathrm{mM}$ HEPES$\mathrm{KOH}, \mathrm{pH} 7.6,10 \mathrm{mM} \mathrm{MgCl} 2$. The chlorophyll content was determined according to Porra and associates (1989).

\section{Blue-native gel electrophoresis (BN-PAGE) and SDS-PAGE.}

BN-PAGE was carried out as described in Kügler and associates (1997) with slight modifications. Thylakoid membranes (equivalent to $20 \mu \mathrm{g}$ of chlorophyll) were washed with $50 \mathrm{mM}$ BisTris- $\mathrm{HCl}, \mathrm{pH}$ 7.0, $330 \mathrm{mM}$ sorbitol, and 250 $\mu \mathrm{g}$ of Pefabloc per $\mathrm{ml}$ (Roche, Mannheim, Germany), were centrifuged at $3,500 \times \mathrm{g}$ for $2 \mathrm{~min}$ at $4^{\circ} \mathrm{C}$, and were resuspended in $25 \mathrm{mM}$ BisTris-HCl, pH 7.0, 20\% (wt/vol) glycerol, and $250 \mu \mathrm{g}$ of Pefabloc per ml. Thylakoids were solubilized with $1 \%$ (wt/vol) n-dodecyl- $\beta$-D-maltoside (Sigma, Steinhaim, Germany) at $0.5 \mathrm{mg}$ of chlorophyll per $\mathrm{ml}$ and, after incubation on ice for $3 \mathrm{~min}$, insoluble material was removed by centrifugation at $18,000 \times \mathrm{g}$ for $15 \mathrm{~min}$. The supernatant was supplemented with $1 / 10$ volume of $5 \%$ Serva blue $\mathrm{G}$ in $100 \mathrm{mM}$ BisTris-HCl, pH 7.0, 0.5 M $\varepsilon$-amino-ncaproic acid (Sigma, Steinheim, Germany), 30\% sucrose and was applied to 5 to $12 \%$ acrylamide gradient gel. The electrophoresis was carried out at $2^{\circ} \mathrm{C}, 100 \mathrm{~V}$ overnight, followed by progressive increases of voltage to $200 \mathrm{~V}$ for $5 \mathrm{~h}$. After the run, excised BN-PAGE lanes were solubilized with $10 \% \beta$-mercaptoethanol in the sample buffer (Laemmli 1970) for $40 \mathrm{~min}$ before the second dimension SDS-PAGE using $15 \%$ acrylamide gels with $6 \mathrm{M}$ urea. After electrophoresis, the gels were silver-stained. 


\section{Western blotting.}

Thylakoids were solubilized in the presence of $6 \mathrm{M}$ urea, and polypeptides were separated by SDS-PAGE using $15 \%$ acrylamide gels with $6 \mathrm{M}$ urea. After electrophoresis, the gels were either stained with Coomassie blue or the proteins were electroblotted to polyvinylidene diflouride membrane, and immunoblot analysis was performed with standard techniques using chemiluminescence for detection. The second antibody was goat antirabbit $\mathrm{IgG}$, alkaline phosphatase conjugated (Zymed, South San Francisco, CA, U.S.A.).

Antisera against different thylakoid proteins have been described earlier (van Wijk et al. 1995). Antisera against TMV $\mathrm{CP}$ was produced in rabbits against purified virions of the wildtype virus.

For production of TMV MP antiserum, the entire coding region of the MP gene of wt TMV (nucleotides 4,903 to 5,709) was cloned in the pET3 vector, expressed in E. coli strain BL21(DE3)pLysE and purified essentially as described by Lehto and associates (1990) and Citovsky and associates (1990), and was used for immunizations. Using this antiserum and the extraction method described by Lehto and associates (1990), the 30-kDa MP was detected from TMV-infected plants with very low nonspecific background.

\section{Quantitation of the chlorophyll content, the chlorophyll $a b$ ratio and the $F_{\mathrm{v}} / F_{\mathrm{m}}$ parameter.}

Chlorophyll content was determined from a leaf disk $(d=1$ $\mathrm{cm}$ ) after incubation in $5 \mathrm{ml}$ of N,N-dimethylformamide in darkness at $4^{\circ} \mathrm{C}$ for $48 \mathrm{~h}$. Chlorophyll extractions and calculation of the amounts of chlorophyll $a$ and $c h l b$ were carried out according to Inskeep and Bloom (1985). The fluorescence parameters and the $F_{v} / F_{m}$ ratio were determined as described earlier (Aro et al. 1993).

\section{Quantitative RT-PCR analysis.}

Quantitative RT-PCR was performed using the Amplifluor Universal Amplification and Detection System (Intergen, Purchase, NY, U.S.A.) as described before (Hiriart et al. 2003). Total RNA extracts, purified as described in Sijen and associates (1996), were cleared of genomic DNA by a 2-h incubation at $37^{\circ} \mathrm{C}$ with RQ1 RNase-Free DNase (Promega, Madison, WI, U.S.A.) followed by phenol extraction. Reverse transcription was performed with the Ready-To-Go You-Prime first-strand beads (Amersham Pharmacia Biotech, Uppsala, Sweden), following the manufacturer's procedures. Typically, $5 \mu \mathrm{g}$ of total RNA was reverse transcribed, and $1 / 5$ of the reaction was used for real-time PCR quantification of target mRNAs. Quantitative PCR reactions $(25 \mu \mathrm{l})$ contained $1 \times$ Thermo-Start Standard Buffer (ABgene, Surrey, England), $1.2 \mathrm{mM} \mathrm{MgCl} 2,250 \mu \mathrm{M}$ dNTPs, 50 $\mathrm{nM}$ Z-tailed specific primer, $500 \mathrm{nM}$ untailed specific primer, $500 \mathrm{nM}$ UniPrimer, and $0.05 \mathrm{U}$ of Thermo-Start DNA polymerase (ABgene) per $\mu$ l. The amplification was done using an ABI Prism 7700 sequence detector system (PE Applied Biosystems, Foster City, CA, U.S.A.), using the following thermocycling program: one cycle of enzyme activation at $95^{\circ} \mathrm{C}$ for 15 min and 50 cycles of template denaturation at $95^{\circ} \mathrm{C}$ for $15 \mathrm{~s}$, primer annealing at $55^{\circ} \mathrm{C}$ for $20 \mathrm{~s}$, and extension at $72^{\circ} \mathrm{C}$ for $40 \mathrm{~s}$.

$\mathrm{ChlH}$ gene quantification was performed with primers already described (Hiriart et al. 2002). For all the other genes, primers have been designed according to sequences previously published for either $N$. benthamiana or $N$. tabacum. To confirm the correct annealing of each primer, their PCR-amplified products were cloned and sequenced. Primer names and sequences are listed below. The Z-sequences are indicated by underlining.

GSA: QGSAREV (5'-GCTGCTAACACCTCATCATCTG-3') and ZQGSAFOR (5'-ACTGAACCTGACCGTACATATTGA CTCCGTGAAGGGCT-3'),
AlaD: QALADFOR (5'-ACCTCTACTGGAGATGAAGCA TACA- $\left.3^{\prime}\right)$ and ZQALADREV (5'-ACTGAACCTGACCGTA CACAGGATATTTGTCTTTCAACAAGCG-3' ${ }^{\prime}$ ),

FeChe: QFcheFOR (5'-CAAGCCTTCGCCTTTTAGAGA G-3') and ZQFcheREV (5'-ACTGAACCTGACCGTACACTT CCCGTTGATACCATGAGG-3'),

ChlD: QChlDFOR (5'CAGACCTTCTTCCCAAGAGC-3') and ZQChIDREV (5'-ACTGAACCTGACCGTACAGTATATT TTACCAGCCACCTCG-3'),

Chll: QChlIFOR (5'-CAATTCAGATCCAGATGACCAA$\left.3^{\prime}\right)$ and ZQChIIREV (5'-ACTGAACCTGACCGTACAGAGC TTTCTCAATGTCGATTGT-3'),

PorB: QPorBFOR (5'-GCGCCAAAGCATACAAAGAC-3') and ZQPorBREV (5'-ACTGAACCTGACCGTACAATGCAG CCAGGGTAAAGAGA-3'),

CS: QCSFOR (5'-GGGACCCCTACACCTGACAT-3') and ZQCSREV (5'-ACTGAACCTGACCGTACAACTGAAGCC CCATTGCTCTA-3'),

25S: Q25SFOR (5'-ACCACAGGGATAACTGGCTTGT-3') and ZQ25SREV (5'-ACTGAACCTGACCGTACACCGACAT CGAAGGATCAAAAAGC-3'),

Lhcbl: QLhcb1REV (5'-AGCCCAAATGGCCAAGAT-3'), and ZQLhcb1FOR (5'-ACTGAACCTGACCGTACAAGCGA GGGTGGACTTGACTACT-3'),

Lhcb2: QLhcb2FOR (5'-CTCAGAAGGCGGTCTTGACTA C-3') and ZQLhcb2REV (5'-ACTGAACCTGACCGTACACT TGGGATGCCCAAATAGC- $\left.3^{\prime}\right)$, and

$P s b A$ : RTpsbaREV (5'-GGTCAAGGAAGTTTTTCTG-3') and ZRTpsbaFOR (5'-ACTGAACCTGACCGTACAGCATAG CACTGAATAGGGA-3').

All sets of primers produce DNA fragments with sizes between 89 and 182 bp.

The relative quantifications were determined from appropriate standard curves prepared from plasmids containing the corresponding target DNA sequence for each gene analyzed. The measured target mRNA amounts were normalizd by relating them to the corresponding 25S RNA levels, amplified from the same total RNA samples and from the same RT-reactions in parallel PCR runs.

\section{ACKNOWLEDGMENTS}

W. O. Dawson is thanked for providing the infectious clone pTMV304. The work has been funded by grants from the Academy of Finland to E.M. Aro and K. Lehto.

\section{LITERATURE CITED}

Abbink, T. E. M., Peart, J. R., Mos, T. N. M., Baulcombe, D. C., Bol, J. F., and Linthorst, J. M. 2002. Silencing of a gene encoding a protein component of the oxygen-evolving complex of photosystem II enhances virus replication in plants. Virology 295:307-319.

Andersson, B., and Aro, E.-M. 2001. Photodamage and D1 protein turnover in photosystem II. Pages 377-393 n: Regulation of Photosynthesis. E.-M. Aro, and B. Andersson, eds. Kluwer Academic Publishers, Dordrecht, The Netherlands.

Aro, E.-M., MacCaffery, S., and Anderson, J. M. 1993. Photoinhibition and D1 protein degradation in peas acclimated to different growth irradiances. Plant Physiol. 103:835-843.

Balachandran, S., Hurry, V. M., Kelley, S. E., Osmond, C. B., Robinson, S. A., Rohozinski, J., Seaton, G. G. R., and Sims, D. A. 1997. Concepts of plant biotic stress. Some insights into the stress physiology of virus-infected plants, from the perspective of photosynthesis. Physiol. Plant. 100:203-213.

Banerjee, N., Wang, J.-Y., and Zaitlin, M. 1995. A single nucleotide change in the coat protein gene of Tobacco mosaic virus is involved in the induction of severe chlorosis. Virology 207:234-239.

Bao, Y., Carter, S. A., and Nelson, R. S. 1996. The 126- and 183- kilodalton proteins of Tobacco mosaic virus, and not their common nucleotide sequence, control mosaic symptom formation in tobacco. J. Virol 70:6378-6383. 
Buchanan, B. B., Gruissem, W., and Jones, R. L. 2000. Page 1367 in: Biochemistry \& Molecular Biology of Plants. American Society of Plant Physiologists. Rockville, MD, U.S.A.

Citovsky, V., Knorr, D., Schuster, G., and Zambryski, P. 1990. The P30 movement protein of Tobacco mosaic virus is a single stranded nucleic acid binding protein. Cell 60:637-647.

Culver, J., Lindbeck, A. G. C., and Dawson, W. O. 1991. Virus-host interactions: Induction of chlorotic and necrotic responses in plants by tobamoviruses. Ann. Rev. Phytopath. 29:193-217.

Dawson, W. O. 1992. Tobamovirus-plant interactions. Virology 186:359367.

Dawson W. O., Beck, D. L., Knorr, D. A., and Grantham, G. L. 1986 cDNA cloning of the complete genome of Tobacco mosaic virus and production of infectious transcripts. Proc. Natl. Acad. Sci. U.S.A. 83:1832-1836.

Dawson, W. O., Bubrick, P., and Grantham, G. L. 1988. Modifications of the tobacco mosaic virus coat protein gene affecting replication, movement, and symptomatology. Phytopathol. 78:783-789.

Esau, K. 1968. Page 225 in: Viruses in Plant Hosts. Form, Distribution and Pathologic Effect. University of Wisconsin Press, London.

Green, B. R., and Durnford, D. G. 1996. The chlorophyll-carotenoid proteins of oxygenic photosynthesis. Ann. Rev. Plant Physiol. Plant Mol. Biol. 47:685-714.

Grimm, B. 1998. Novel insights in the control of tetrapyrrole metabolisms of higher plants. Curr. Opin. Plant Biol. 1:245-250.

Hiriart, J. B., Lehto, K. Tyystjärvi, E., Junttila, T., and Aro, E.-M. 2002. Suppression of a key gene involved in chlorophyll biosynthesis by means of virus-inducing gene silencing. Plant Mol. Biol. 50:213-224.

Hiriart, J.-B., Aro, E.-M., and Lehto, K. 2003. Dynamics of the VIGSmediated chimeric silencing of the Nicotiana benthamiana ChlH gene and of the tobacco mosaic virus vector. Mol. Plant-Microbe Interact. 16:99-106.

Hodgson, R. A. J., Beachy, R. N., and Pakrasi, H. B. 1989. Selective inhibition of photosystem II in spinach by Tobacco mosaic virus: An effect of the viral coat protein. FEBS (Fed. Eur. Biochem. Soc.) Lett. 245:267-270.

Inskeep, W., and Bloom, P. R. 1985. Extinction coefficients of chlorophyll $a$ and $b$ in N,N-dimethylformamide and $80 \%$ acetone. Plant Physiol. 77: 483-485

Itaya, A., Matsuda, Y., Gonzales, R. A., Nelson, R. S., and Ding, B. 2002. Potato spindle tuber viroid strains of different pathogenicity induces and suppresses expression of common and unique genes in infected tomato. Mol. Plant-Microbe Interact. 15:990-999.

Jarvis, P., and Soll, J. 2001. Toc, Tic, and chloroplast protein import. Biochim. Biophys. Acta 1541:64-79.

Jockusch, H., Wiegand, C., Mersch, B., and Rajes, D. 2001. Mutants of Tobacco mosaic virus with temperature-sensitive coat proteins induce heat shock response in tobacco leaves. Mol. Plant-Microbe Interact. 14:914-917.

Kügler, M., Jänsch, L., Kruft, V., Schmitz, U. K., and Braun, H.-P. 1997. Analysis of the chloroplast protein complexes by blue-native polyacrylamide gel electrophoresis (BN-PAGE). Photosynth. Res. 53:3544.

Laemmli, U. K. 1970. Cleavage of structural proteins during the assembly of the head of bacteriophage T4. Nature 227:680-685.

Lehto, K., Bubrick, P., and Dawson, W. O. 1990. Time course of TMV $30 \mathrm{~K}$ protein accumulation in intact leaves. Virology 174:290-293.

Lewandowski, D. J., and Dawson, W. O. 1993. A single amino acid change in tobacco mosaic virus replicase prevents symptom production. Mol. Plant-Microbe Interact. 6:157-160.

Lindbeck, A. G. C., Dawson, W. O., and Thomson, W. W. 1991. Coat protein-related polypeptides from in vitro tobacco mosaic virus coat protein mutants do not accumulate in the chloroplasts of directly inoculated leaves. Mol. Plant-Microbe Interact. 4:89-94.

Lindbeck, A. G. C., Lewandowski, D. J., Culver, J. N., Thomson, W. W., and Dawson, W. O. 1992. Mutant coat protein of Tobacco mosaic virus induces acute chlorosis in expanded and developing tobacco leaves. Mol. Plant-Microbe Interact. 5:235-241.

Melchers, G. 1942. Uber einige Mutationen des Tabakmosaicvirus und eine "Parallelmutation" des Tomatenmosaikvirus. Naturwissenschaften $30: 48$.

Moore, C. J., Sutherland, P. W., Forster, R. L. S., Gardner, R. C., and MacDiarmid, R. M. 2001. Dark green islands in plant virus infection are the result of posttranscriptional gene silencing. Mol. Plant-Microbe Interact. 14:939-946.

Oxelfelt, P. 1970. Development of systemic tobacco mosaic virus infection. I. Initiation of infection and time course of virus multiplication. Phytopath. Z. 69:202-211.

Oxelfelt, P. 1971. Development of systemic tobacco mosaic virus infection. II. RNA metabolism in systemically infected leaves. Phytopath Z. 71:247-256

Porra, R. J., Thompson, W.A., and Kriedemann, P. E. 1989. Determination of accurate extinction coefficients and simultaneous equations for assaying chlorophyll $a$ and $b$ with four different solvents: Verification of the concentration of chlorophyll by atomic absorption spectroscopy. Biochim. Biophys. Acta 975:384-394.

Rahoutei, J., Garcia-Luque, I., and Baron, M. 2000. Inhibition of photosynthesis by viral infection: Effect on PSII structure and function. Physiol. Plant. 110:286-292.

Reinero, A., and Beachy, R. N. 1986. Association of TMV coat protein with chloroplast membranes in virus-infected leaves. Plant Mol. Biol. 6:291-301.

Reinero, A., and Beachy, R. N. 1989. Reduced photosystem II activity and accumulation of viral coat protein in chloroplasts of leaves infected with Tobacco mosaic virus. Plant Physiol. 89:111-116.

Shintaku, M. H., Carter, S. A., Bao, Y., and Nelson, R. S. 1996. Mapping nucleotides in the $126-\mathrm{kDa}$ protein gene that control the differential symptoms induced by two strains of Tobacco mosaic virus. Virology 221:218-225.

Shivprasad, S., Pogue, G. P., Lewandowski, D. J., Hidalgo, J., Donson, J., Grill, L. K., and Dawson, W. O. 1999. Heterologous sequences greatly affect foreign gene expression in tobacco mosaic virus-based vectors Virology 255: 312-323.

Sijen, T., Wellink, J., Hiriart, J-B., and van Kammen. 1996. RNA-mediated virus resistance: Role of repeated transgenes and delineation of targeted regions. Plant Cell 8: 2277-2294.

Suorsa, M., Zhang, L., Battchikova, N., Paakkarinen, V., Regel, R., Herrmann, R., and Aro, E.-M. 2001. Assembly of photosystem II in chloroplast psbEFLJ operon mutants. In CD-Rom: PS2001 Proceedings: 12th International Congress of Photosynthesis. CSIRO Publishing, Melbourne, Australia.

van Kooten, O., Meurs, C., and van Loon, L. C. 1990. Photosynthetic electron transport in tobacco leaves infected with Tobacco mosaic virus. Physiol. Plant. 80:446-452.

van Wijk, K. J., Bingsmark, S., Aro, E.-M., and Andersson, B. 1995. In vitro synthesis and assembly of Photosystem II core proteins. The D1 protein can be incorporated into Photosystem II in isolated chloroplasts and thylakoids. J. Biol. Chem. 270:25685-25695.

von Wettstein, D., Gough, S., and Kannangara, C. G. 1995. Chlorophyll biosynthesis. Plant Cell 7:1039-1057.

Zhang, L., and Aro, E.-M. 2002. Synthesis, membrane insertion and assembly of the chloroplast-encoded D1 protein into Photosystem II. FEBS (Fed. Eur. Biochem. Soc.) Lett. 512:13-18.

Zhang, L., Paakkarinen, V., van Wijk, K. J., and Aro, E.- M. 1999. Cotranslational assembly of the D1 protein into photosystem II complex. J. Biol. Chem. 274: 16062-16067.

Zhang, L., Paakkarinen, V., van Wijk, K. J., and Aro, E.-M. 2000. Biogenesis of chloroplast-encoded D1 protein: Regulation of translational elongation, insertion and assembly into photosystem II. Plant Cell 12:1769-1782. 